\title{
Understanding Unstable Information Systems Phenomena: A Punctuated Equilibrium Perspective
}

\author{
Robert E. Crossler \\ Washington State University \\ rob.crossler@wsu.edu
}

\author{
France Belanger \\ Virginia Tech \\ belanger@vt.edu
}

\author{
Carlos I. Torres \\ Washington State University \\ carlos.torres@wsu.edu
}

\author{
Allen Johnston \\ University of Alabama \\ ajohnston@.cba.ua.edu
}

\author{
Merrill Warkentin \\ Mississippi State University \\ m.warkentin@msstate.edu
}

\begin{abstract}
The information systems (IS) literature includes different perspectives, epistemologies, and research philosophies to explore phenomena at the intersection of technologies, information, people, organizations, and processes. As studies are replicated and knowledge accumulates, researchers can develop a more in-depth understanding of how their constructs of interest interact and affect each other. IS researchers have reported mixed findings in prior research as the phenomena change. In this paper, we discuss unstable phenomena in IS and argue that conflicting findings in a variety of domains might be the result of this instability. Using examples from IS security and word processing research streams, we examine the issues surrounding unstable phenomena using a punctuated equilibrium lens and suggest research strategies and a research framework to help researchers conduct studies in this challenging environment.
\end{abstract}

\section{Introduction}

Information systems (IS) researchers study a variety of phenomena, often focusing on the interactions between technologies, information, people, organizations, and processes. However, during the systemic process of an IS, changes in the interactions of different components in a system, can affect systemrelated organizational outcomes [1]. As the role of change in a system is coupled with the various topics covered by IS research, opportunities for further theoretical understanding present themselves. The important role of "change" in IS research can be identified from 1985 to 2006 and its five core research areas: information technology and organizations, IS development, IT and individuals, IT and markets, and IT and groups [2]. Sidorova et al. suggest that whereas the core topics have remained relatively stable over time, the specific research themes within each area "have evolved significantly... [with research] ... focused less on technology development and more on the social context in which information technologies are designed and used" [2] (p. 467). Another study reviewed over 1,800 articles from 2010 to 2015 to suggest that IS research is cyclical as it adapts from changes in the research environment [3] . All these authors highlight that over time there is increased complexity of topics studied with the pace of change also increasing substantially. These studies, thus, further reinforce the idea that IS researchers explore phenomena that constantly evolve over time. Though the field of IS continues to grow and accumulate knowledge, researchers continue to highlight the ontological changes in the phenomena studied by IS scholars, thus creating new challenges and conflicting findings [4]. For example, mixed findings have been identified in the study of information security [5, 6], e-government [7], and computer self-efficacy $[8,9]$, to name a few.

Even when IS researchers use the same theoretical foundation to study similar constructs, the phenomena continue to change, thus offering mixed findings [e.g., $5,6]$. Those differences may be explained by different types of changes; e.g., from a physical object to a digital object, to a place where digital technologies are now creating and shaping physical reality (e.g. videos, news, documents, software, tickets and even money) [4, 10]. Thus, the questions of interest is: what leads to changes in phenomena and how do these changes generate inconsistent findings? Possible explanations previously suggested include divergent approaches [e.g., 7, 11] and divergent philosophies or applications of theories [e.g., $6,11]$. In this article, we argue that another potential explanation for inconsistent findings in prior research is the unstable (i.e., changing) nature of the IS concepts that are explored.

A phenomenon is said to be unstable if it is characterized by dynamic, unpredictable factors, relationships, and events that challenge our ability to establish contextual clarity, valid measurements, and reliable methods. Consider Truex, Holmström and Keil 
[12]'s first criterion for theory adaptation, whereby scholars contemplate the fit between a selected theory and a phenomenon of interest. This fit is a critical early footing for researching a phenomenon and is highly dependent upon the researcher's access to the field, to the specific contextual attributes from which a phenomenon has taken root, and to a thorough understanding of the contextual attributes, factors, and relationships that shape the phenomenon. For unstable phenomena, however, these elements are constantly evolving, emerging, or regressing, and our understanding of them is temporary in nature and frequently deficient in detail. Therefore, the "fit" is made more difficult in circumstances in which "facts" concerning a particular phenomenon are increasingly allusive, as is the case with unstable phenomena. Unstable phenomena create unique challenges in that solely positivist, cross-sectional, or quantitative approaches likely result in missing or misconceived measures of the phenomenon and its contextual attributes. Researchers may struggle to understand an unstable phenomenon, which may lead them to misapply theory or misrepresent observations. Furthermore, to better build upon prior work and perform proper theorizing, researchers need to be able to identify the relative stability or instability of the phenomenon of interest.

In this paper, we draw on equilibrium theory [13] to demonstrate the instability of some IS phenomena and explain the consequences of instability, we then propose a punctuated equilibrium perspective to understand instability with IS phenomena examples. Finally, we propose a framework to help deal with IS phenomena instability. We argue that it is under equilibrium that researchers can develop a complete conceptualization of the phenomenon to adapt theory that fits it. In contrast, during periods of instability (punctuations), theories may not adjust or represent the phenomenon entirely or could even misrepresent it based on the characteristics of the punctuation. Even though the phenomenon under study has evolved, becoming more digitally facilitated, it will reach an equilibrium (stability phase) and research will thrive based on what is learned at different equilibrium stages.

\section{Phenomena Instability in IS Research}

To study IS phenomena, researchers bring together natural and the social sciences [14] - evaluating the relationships between technologies, information, people, organizations, and processes on one hand and the design, implementation, adoption, use, and impacts of information technology (IT) artifacts on the other.

Integrated circuits and algorithms may be predictable, but users and managers are not. The IS discipline seeks to understand processes and relationships that are not always well understood or easily fully conceptualized. Further, a phenomenon that is understood at one time within a specific context may morph into a very different system that must be reassessed at another time, and the theories or measures that once provided insights may no longer be reliable when attitudes, values, perceptions, habits, and beliefs change. When compared to many other business disciplines, the IS field appears more dynamic; "Accounting, finance, and other business school disciplines traditionally serviced much more stable constituencies than IS" [15 p. 140]. However, we need to recognize that these disciplines have in recent years experienced some instability linked to the disruptive nature of technology. In fact, over time, it is this sometimes disruptive nature of technology that has forced IS researchers to adapt to a moving target of constituents and the artifacts that they study. This started with research into the use of IBM programming languages, to decision support systems, to microcomputer systems, and later to e-commerce and organizational systems [15]. This adaptation to moving targets within IS research was also highlighted by Applegate [16] who laid out five eras of computing, each with its own set of technologies, applications, and research issues. In fact, in information systems, there seems to be a continuing state of change [17].

Baskerville and Myers [18] provided a possible explanation for these moving targets by stating that IS research follows fashion waves. They reviewed articles in four IS research domains (office automation, computer-aided software engineering tools, business process reengineering, and electronic commerce), and suggested that there are fashion upswings and fashion downswings. They concluded that in downswings researchers continue to explore areas that are no longer of interest to practitioners. The idea that IS researchers follow "fashion waves" was debated by Gill and Bhattacherjee [19] who argued that their informing model might better explain the cycles IS research follows for each phenomenon of interest. They suggested that technologies move from novelty to institutionalization, and that interest fades as the topics become heavily researched and there are fewer "interesting propositions" to study. More recently, Cram, Brohman and Gallupe [20] suggest that moving targets are common in IS implementations, and that through controls and control change processes, the instability can be regulated. Contrary to this perspective, we argue that the relative instability of IS phenomena will continue to exist and might explain differences in findings that are reported over time. This suggests that studies are needed at multiple points in time of a phenomenon's existence and that researchers need to evaluate the relative state of instability of the phenomenon of interest in their research. 


\section{Punctuated Equilibrium and Instability}

The above discussion establishes that the nature of IS research is one that is regularly addressing phenomena instability. This phenomena instability can be explained through a process called punctuated equilibrium [13]. Punctuations are a fundamental factor in a process theory to explain information system changes[1]. In a Punctuated Socio-Technical Information System Change process model, the theory explains the effect that punctuations in multiple levels within a system can have on organizational outcomes [1], by focusing on understanding the change in a process. Our paper, in contrast intends to study the effects of change in affecting relationships among variables in variance theories. Punctuated equilibrium theory has also been used to explain value creation in a supply chain IT system process [21], where again the focus is in the process and not in the relationships. We argue in this paper that the process of digitalization follows the same punctuated equilibrium pattern.

As presented in Figure 1, punctuated equilibrium is a process in which a phenomenon undergoes evolutionary periods of little change (or equilibrium) and then revolutionary periods of transformational change, resulting in instability [22]. This period of change is the punctuated portion of the equilibrium, in which the phenomenon is in such a state of flux that our understanding of it also changes. During periods of equilibrium, it is possible to gain a more complete understanding of the phenomenon and to develop theoretical foundations that readily describe the phenomenon. During the periods of punctuation, the phenomenon is in a state of upheaval such that gaining a theoretical understanding from a variance perspective of that phenomenon is difficult and elusive. Utilizing the punctuated equilibrium lens to understand different phenomena is especially insightful as passing through a revolutionary period results in a new evolutionary period where the new state does not assume that the previous states are universally followed [22]. This opens a new opportunity for theoretical understanding of the new evolutionary period.

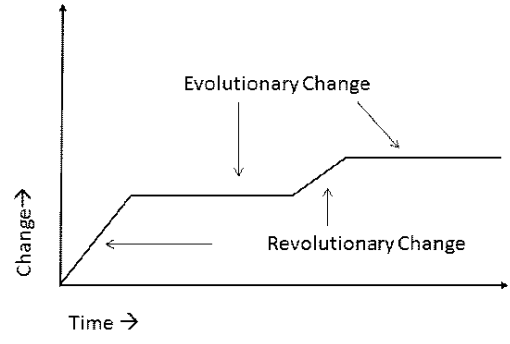

Figure 1. Punctuated Equilibrium

By considering the impact of punctuations on phenomena, researchers can select which research tools they want to use to answer a given research question. In particular, looking at research questions through a punctuated equilibrium lens provides a long-term focus on the research of interest [22]. If the assumption is that the stability of a phenomenon is always constant, researchers may not be sensitive to changes in context that would affect a given study. However, by being attuned to the current state of the phenomenon and changes that occur to it, appropriate decisions can be made regarding theoretical foundations and research designs that further researchers' understanding in ways not possible without consideration of this contextual difference. Comprehending the changing nature of a phenomenon can also help researchers design more thorough and complete research programs that attempt to shed light on the phenomenon of interest over time.

The punctuated equilibrium can also help explain mixed findings in prior research as phenomena change due to an increase in digitization with more and more functionality relying on Internet connectivity. To demonstrate this, we explore two domains of IS research where instability has been demonstrated over the years: word processing and information security (InfoSec). The word processing example is used to illustrate how instability caused by digitalization has been pervasive in the IS domain for a long time. Although it is unlikely that most IS researchers would think of word processing as an unstable phenomenon, its history shows otherwise. This makes PC-based word processing a powerful research domain to illustrate periods of instability with the punctuated equilibrium framework. It should be noted that in this discussion of instability, we will not consider pre-personal computer word processing, which took the form of special-purpose office machines or typewriters [23]. The example of InfoSec will later provide a more in-depth discussion of the issues that arise when dealing with unstable phenomena.

\subsection{Instability in Word Processing}

Word processing for the Personal Computer (PC) has its origins in the middle of the 1970s, and is considered one of the most significant PC applications (together with spreadsheets) [23]. Indeed, word processing and spreadsheets were the original "killer apps" that drove computer sales for years. In recognition of this, the IEEE Annals of the History of Computing published a special issue dedicated to word processing in 2006. We discuss various periods of instability and stability of the word processing domain below.

Unstable beginnings: Hardware dependencies and incompatibilities. The initial application, Electric Pencil, required that each copy be configured for the user's specific computer. Initially written in 8080 assembler in 1976, it later had eight versions for different hardware configurations [24]. At almost the same time (1977), the Apple II was revealed and the 
word processor EasyWriter was written for it in Assembly language [24]. As a result of the nonstandard disk operating system, it had files incompatible with other software. It was then prematurely ported to the IBM PC before full development and testing, which resulted in its downfall [24]. The third major player in the early word processing market was WordStar by MicroPro (1979). Also written in 8080 assembler, it was difficult to port to other operating systems. Versions available for other platforms did not allow users to transfer their files to the new format easily [24].

In summary, the early years of word processing showed significant instability, with dependencies on hardware. By 1984, there were over 300 word processing software on the market, with concomitant technical incompatibilities, hardware dependencies, and user difficulties to migrate from one software to another.

Relative Stability. A major event that created some stability in the world of word processing was the acceptance of the IBM PC (introduced in 1981) and its operating system MS-DOS by the mid-1980s, which led to a "more homogenized market" [24, p. 43]. At the same time, word processing software evolved in the mid-80s to contain standard printer support, tutorials, and user manuals. There was also an attempt by developers to provide compatibilities across several of their own platforms [25]. There were still a large number of software providers for word processing, but given the preferred platform of MS-DOS for the IBM PC, there were just a few dominant players: WordPerfect and Microsoft Word [25]. This led to a stable era until the next major innovations: WYSIWYG (What You See Is What You Get) and Windows.

Instability: Computing Platforms. The idea of WYSIWYG for word processing became prevalent after the announcement of the OS/2 and its presentation manager in 1987 [25]. In a few years of relatively stable development and use of word processing, the need arose to have better graphical interfaces. At the same time, Windows grew as the leading operating system. Because WordPerfect initially failed to include WYSIWYG and to participate in the development of their product for the Windows platform, they lost their leadership position in the word processing market [25]. Word, on the other hand, which was also released in the mid-1980s, offered some new concepts such as the use of a mouse together with the implementation of many desired features (standard printer databases, etc.). By 1987, with Word 4.0, it had already implemented the desired WYSIWYG [25]. By 1985, there was also a hardware-independent Word version (e.g., Macintosh and Windows environments). This ability to use similar software in the two platforms, and eventually to port documents between the two platforms, led to its dominance, which lasted from the late 1980s to the late 2000s. This was a very long period of stability, which would lead us to think that word processing is a stable phenomenon. Yet, a new concept then changed the world of word processing again.

New Instability: Shared Writing. In the mid2000s, a new combination of technology innovation and user requirements led to a new punctuation, that of collaborative cloud-based software. For example, Google Docs allowed users to not only use a web interface to write documents, but also share documents and collaboratively write with others. This innovation has evolved with many of such software products available. With collaborative cloud-based software, users had to re-learn how to write documents in consideration of their co-writers, making sure not to remove needed material from shared folders.

In summary, the history of PC-based word processing shows that this well-established computing domain has shown and continues to show periods of instability, as summarized in Figure 2.

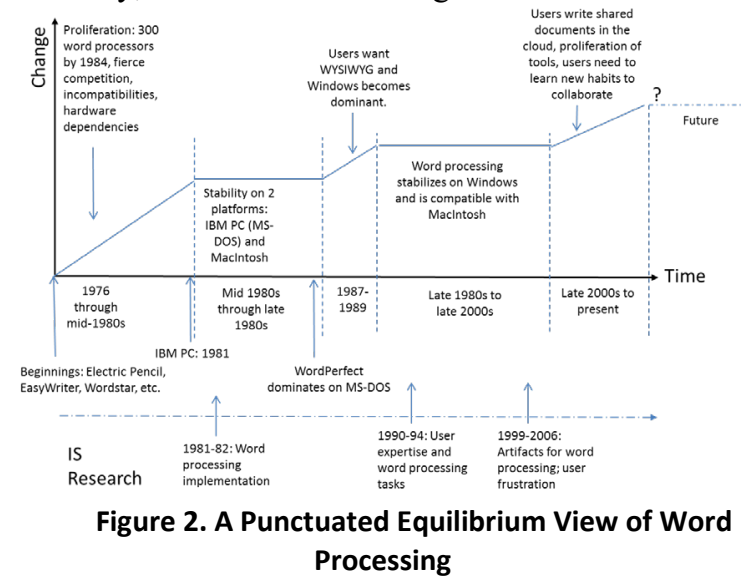

From a research standpoint, studies of word processing followed some of the same transitions as the implementation of word processing did, although the research has yet to be widely published on cloud-based shared writing, and is mostly available in fields outside of IS [26] or in conference proceedings [e.g., 27]. In the early days of word processing, two papers were published in MIS Quarterly. The first discussed the integration of word processing within data processing [28]. It explained how word processing impacted the flow of information and managerial roles. The other discussed the process of selecting and implementing a word processing system in the late 1970s [29]. Then, few research papers were published in journals until the early 1990s, when some studies focused on novice versus experienced users. At this time, further research also investigated whether computer-based writing helped learning [30]. Another area of research focused on the tools such as spell checkers [31].

By the late 1990s and early 2000s, most research took the form of designing or evaluating word processing artifacts, such as the development of 
interactive spell checkers [32] and Add-Ins [33]. Some researchers also studied users and word processing, specifically focusing on user frustration [34, 35], while other research studied the pricing of word processing software [36]. This brief review is consistent with Baskerville and Myers' [18] observation that research on office automation peaked in 1984 and then dwindled.

\subsection{Instability in Information Security}

Information Security (InfoSec) presents another example where researchers have assessed sociotechnical phenomena characterized by instability, with the added issue of more sources of instability. InfoSec involves the study of behavioral and technical threats to IS and the responses or solutions to such threats for protecting information. The domain includes many rapidly evolving and unstable phenomena [17]. This instability is forced in part by rapid technological, social, and managerial advancements, but also in part by the deliberate counterforce working against its stability. Hackers, identity thieves, and others actively and systematically alter the threat vectors - their processes and behaviors, their methods of attack, and their reaction to attempt to deter or prevent their attacks [37]. In fact, obfuscation is a standard principle of most attacks [38]. Once researchers theoretically understand what causes users' behaviors, the attackers (also knowing this) change their attack vectors. This issue represents an unstable phenomenon that cannot be fully captured in variance models where the action-reaction just discussed cannot be captured over time.

While many domains within InfoSec follow this cycle of actions-reactions, we turn to the specific InfoSec area of malware for illustration purposes. As can be seen in Figure 3, computer viruses first began to propagate in the 1970s [39]. This was followed by a season of stability concurrent with the widespread availability of anti-virus software. This IT artifact provided better protection, leading to a relative period of stability. However, as high-speed Internet access became widely available, so did individuals' continuous connection and the increased apparition of selfpropagating worms [40]. After this transition period, as anti-virus providers worked hard to develop solutions, the availability of automated tools removed the human factor to some degree from the equation. Anti-virus software would now automatically update by default and users would be warned if their computer did not have that feature turned on. This automation resulted in another period of relative phenomenon stability.

The malware phenomenon entered another season of instability as hackers upped their game and found additional vectors to beat anti-virus defenses. One example of this was found in the source code theft of one of Symantec's anti-virus tools. This resulted in Symantec encouraging people to stop using a particular version of their anti-virus software and recommending that customers utilize security countermeasures from a number of vendors instead of a suite from a single vendor [41]. This recommendation changed individual behavior, but whether or not it is significant enough to cause a period of instability will be determined by how well anti-virus software makers adapt to combat this escalating arms race. Another reason for a possible new punctuation is the rise of polymorphic viruses, which can self-modify to avoid detection by anti-virus software as they are propagated through systems and networks. Furthermore, recent malware includes the infection of machines with ransomware. Ransomware encrypts the data on an infected machine and requires the user to pay ransom, generally in the form of bitcoins, in order to receive the key to unlock the encrypted files. Due to the adaptation of hackers to anti-malware protection, ransomware has spread in ways that are not entirely protected software. Recommendations like keeping systems patched and not clicking on links in emails [42] require increased effort by individuals, such that existing theoretical models of user behaviors may also need to be reevaluated.

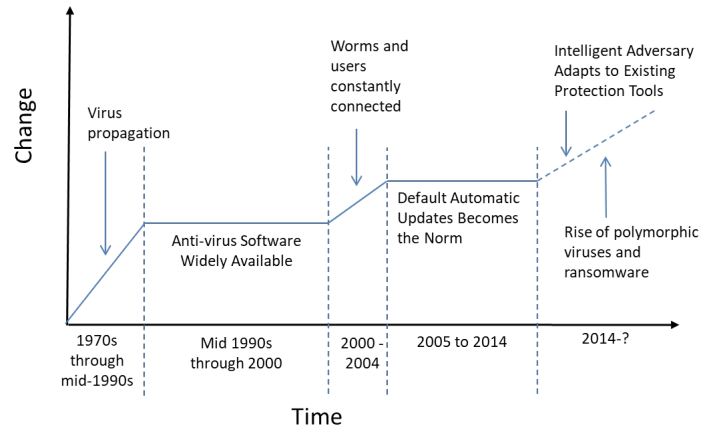

Figure 3. Punctuated Equilibrium of Malware

Instability for InfoSec phenomena has impeded the research community's efforts to reliably apply and adapt theories toward the investigation of the phenomenon, resulting in the stagnation of theory development and advancement within the domain when approached from the predominant positivistic perspective. The adaptation of Protection Motivation Theory (PMT) within the InfoSec literature is representative of the difficulties facing IS scholars. The progression and refinement of PMT within the InfoSec research domain remains evasive although it has been widely applied to understand protective behaviors adopted by users [e.g., 5, 11, 43, 44-46]. PMT suggests that individuals go through a threat and coping appraisal process to determine whether to adaptively cope with the threat by performing a recommended behavior. The threat appraisal process includes their assessment of the severity of and vulnerability to the threat. The coping appraisal process includes an assessment of their ability to perform the recommended behavior, the effectiveness of the behavior at mitigating the threat, and the 
perceived costs of performing the behavior. Studies using PMT have had mixed results with respect to the proposed theoretical relationships. For example, in some studies the threat appraisal construct of vulnerability and the coping appraisal construct of response cost were found to have a significant impact on the dependent variable as hypothesized [46]. In other studies, however, at least one of these constructs was not significant in the determination of protection motivation [43-45]. One possible explanation is that each of these studies performed their own adaptation of the base PMT theory [11]. It is also possible that these studies explored InfoSec protection behaviors that were unstable at the time of the study. Without a proper immersion into the context of the phenomenon, it is possible that some of the studies did not include relevant factors or relationships that would have helped explain the targeted behaviors. Recall that when a phenomenon is unstable it is likely that there will be unpredictable factors, relationships and events that challenge the researchers' ability to provide contextual clarity, and valid and reliable measurements. Our proposed framework presented later details how to perform phenomenon immersion and the role of theory adaptation in handling unstable phenomena.

\section{Framework For Researching Unstable IS Phenomena}

The examples presented above demonstrate that some IS phenomena exhibit instability at some point in time. The causes of instability are multiple, although all related to the rapid process of digitalization; in the case of word processing, it is a combination of platform changes, changes in user experiences, and increased demands for features by users. For InfoSec, causes of instability include improvements in artifacts, automation, user behaviors, as well as constantly evolving counterattacks (hackers, malware, hacking tools, etc.). The examples also demonstrate how instability can impact theory selection and adaptation. It is therefore important for IS researchers to identify the relative stability or instability of the phenomenon of interest in order to better build upon prior work and perform proper theorizing given these causes.

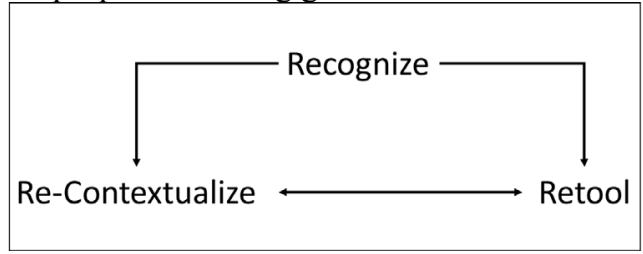

Figure 4. R3 Framework for Understanding Unstable IS Phenomena

By understanding the nature of instability in IS phenomena, researchers may be better positioned to advance knowledge on the given phenomenon by building on prior findings and following sound scientific principles. To provide a steppingstone in that direction, we propose the following high-level R3 framework to consider unstable IS phenomena: recognize, re-contextualize, and retool. This framework is illustrated in Figure 4.

\subsection{R1: Recognize}

The first step necessary to study an unstable IS phenomenon begins with recognizing that the phenomenon has entered a stage of instability. Punctuated equilibrium provides a basis to recognize when changes have occurred to the baseline of the phenomenon, resulting in changing its state to unstable.

To recognize instability, IS researchers must immerse themselves in each investigated phenomenon so that they are in tune with their contexts, the factors at play, and the relationships among those factors. According to Johns [47, p. 386], context refers to "situational opportunities and constraints that affect the occurrence and meaning of organizational behavior as well as functional relationships between variables." As scholars, we often observe phenomena from the fringes, which limits our ability to see the nuances that shape behaviors and interactions between technology and people. Further, with greater phenomenon immersion, researchers could more readily identify relatively stable factors, as observed in the word processing literature. Identifying when phenomena are in a state of instability requires studying the 'what' of the phenomenon's context as opposed to trying to move directly into prediction [48]. When differences begin to emerge in explaining what is going on with a phenomenon, the ability to predict that phenomenon diminishes. This is illustrative of a punctuation in the equilibrium assumed in prior theory. Successful implementation of word processors reached a point where success no longer required advanced technological capabilities, but rather required user reactions to the technology. As word processing technologies moved towards cloud-based collaborative word processing, a new state of punctuation arose that must address the group-share context of the technology.

There are different ways for researchers to immerse themselves into a phenomenon to identify how unstable it may be. IS researchers have used ethnographic studies in a traditional anthropological way to observe a phenomenon by being part of it, but without exercising any type of actions that can modify the environment under study [49]. Other more active qualitative methods such as action research or design ethnography suited for IS research [49]. Others suggest immersion via a deep and precise phenomenon-oriented literature review that allows researchers to identify the phenomenon's historical context [50]. 
As researchers struggle to understand an unstable phenomenon, they may misapply theory or misrepresent observations. To avoid these validity concerns, researchers need to pay attention to possible punctuations while developing their literature reviews. We recommend that researchers look for states in which a phenomenon might have evolved and the factors that have led to punctuations. In doing so, they can reconcile conflicting findings and better consider the assumptions inherent in the research questions they develop. When researchers recognize the existence of instability, the various evolving states of the phenomenon, and punctuations, they are better equipped to contextualize their research and conduct their studies.

IS researchers can also draw from management research where there are many discussions of the ways that organizations recognize and take advantage of a punctuation by considering when a punctuation is emerging and selecting the strategy to follow. A possible punctuation is recognized through the increasing complexity of tasks or through environmental turbulence; either leads to change of state from stable to unstable, or vice-versa [51]. Researchers would have spotted punctuation in the word processing and technology adoption discussion above through the presence of an increased complexity. The information security punctuation would have been identified through environment turbulence within the phenomenon. Once theorists identify the emerging punctuation then they can move on to the second step of the R3 framework.

\subsection{R2: Re-contextualize}

When the phase of stability or instability of a focal phenomenon has been recognized, a process of recontextualizing the theoretical foundations, hypotheses and models needs to occur. As previously stated, context refers to opportunities and constraints of a particular situation, which can affect behavior and relationships between variables of interest [47]. Within IS, this context is captured by considering the characteristics and usage of technological artifacts $[52,53]$ and its process of digitalization or if the artifact is entirely a digital object with no physical representation $[4,10]$. When a phenomenon is undergoing punctuation, the situations and constraints that were once understood to affect that phenomenon are in a state of change. As such, part of re-contextualizing requires understanding what these new situations and constraints are and positioning theory within these contexts. Through this process, constructs may expand or narrow in their definition over time as relationships in nomological nets are uncovered.

As a phenomenon is undergoing punctuation, there is further influence on theory depending on the role that the IS artifact plays within that theory. The IT artifact or the digital object is not adequately captured in the vast majority of IS research and theories even though the artifact is an important element within IS research that bridges the social and information domains with the technological phenomena being studied [e.g., 10, 53]. As these phenomena are changing, it is important to recontextualize the theories and constructs being used to determine how the technology changes concerning what the theory is explaining. This could be done by linking the changing nature of the phenomenon of interest with the constructs utilized in various theories [52]. By considering the role of changed phenomenon and technology in the theory, the understanding of the information system can be more accurately captured. For example, as one considers the changes that have occurred in research related to malware, it is evident that the role of technology has changed over time. Research in this area began with a semblance to adoption literature focusing on whether or not people intended to utilize appropriate technical tools to protect their computer [e.g., 45, 46, 54]. As the complexity of vulnerabilities changed, this approach moved to an investigation of the individuals and how they make decisions related to falling victim to social engineering attacks [55]. The change in the role of technology in influencing how individuals' information becomes vulnerable was caused by punctuation in the phenomenon and required a change in how researchers studied malware. There is now a need for a new theory to begin to understand how to deal with constantly evolving threats, both human and technical, which represents the latest malware punctuation. It may be that researchers need to develop Type 1 theories [56] to better analyze how constantly changing vectors of attack influence counter-reactions to malware before researchers can again explain or predict the relationships between constructs of interest in the malware domain (i.e., Types 2 and 4 theories [56]).

\subsection{R3: Re-tool}

The need to retool for unstable IS phenomena results from the necessity to recognize the phases of instability of the phenomenon being studied. Once the appropriate theory is identified to understand a changed phenomenon retooling can involve developing new instruments that correctly capture the constructs' underlying factors [57]. Retooling also suggests researchers need to engage more in-depth with the phenomenon. As previously discussed, phenomena in a state of instability create unique challenges in that solely positivist, cross-sectional, or quantitative approaches may not be sufficient to truly contextualize a study. This lack of contextualization can lead to missing key factors and/or relationships and to misconceived measures of the phenomenon and its contextual attributes.

While research on phenomena at a point of stability can see the prevalence of Type 4 theories (theories for 
explaining and predicting) [56], punctuations require revisiting of the core assumptions and context of the phenomenon of interest. One approach previously mentioned to do this is phenomenon immersion, which can occur through the use of various methodological approaches. Phenomenon immersion means being deeply involved with the phenomenon of interest. We have mentioned multiples ways of involving deeply with the phenomenon. When researchers are in exploration mode, they should be open to the idea that theories that once worked can be challenged as the phenomenon they seek to explain may be misunderstood and continue to evolve. At this stage, exploratory research that uses methods allowing inductive reasoning based on observations of the phenomenon can help understand its relative phase of stability or instability. This can be followed by a period of evolutionary change, when explanatory research improves on prior theoretical foundations to deduce conclusions based on those theories.

The suggestion to use multi-method research approaches, combing several qualitative and quantitative research methodologies, is not new. However, in considering the series of punctuations and periods of stability of IS phenomena, what we are suggesting is that methodologies are combined not necessarily in one study but in multiple studies over a longer period of time. Word processing research exemplifies this approach since research occurred in bursts with long gaps in published research regarding this phenomenon. Each new burst of research represented a new change in the word processing environment and was discussed utilizing different methodologies. During the first phase of research in word processing, the research utilized a qualitative methodology. By taking this approach, researchers were able to fully understand this newly emerging phenomenon and, through the process of a qualitative analysis, apply Mintzberg's theoretical concept of managerial roles and information flows [28]. The second era of word processing literature occurred after the word processing environment had undergone rapid changes in the 1980s and into the 1990s. When this research stream emerged again, researchers began utilizing experimental methods to understand this phenomenon. In particular, an experimental approach was used to determine the level of specificity that needs to be provided in teaching users to use word processing software [58]. Another study also utilized an experimental approach to determine that intermittent users of word processing software acted like experts in the small number of tasks they knew, but novices in most other tasks. It was further shown that intermittent users preferred a menu driven interface and the presence of a help system [59].
Later research utilized several methods to focus on technologically improving and evaluating advances in the development of word processing software. One study utilized a case study, developed through the process of interviewing developers of a Japanese language word processor. The interview process enabled the authors to uncover factors that led to the successful development of the software [60]. In another study, an experiment was performed to help test advances made in the development of a new spell checker [32]. A qualitative analysis of users' time diaries of their use of word processing software resulted in identifying factors that led to user frustrations of using these systems $[34,35]$. In addition, an economics analysis utilized a hedonic approach to understand pricing structures for word processing software [36]. As exemplified within the word processing research, the utilization of several different research methods provided immersion into this phenomenon that one methodological approach alone could not provide.

Traditional methods of engagement such as ethnographies or phenomena-based literature reviews are excellent ways to immerse into the phenomenon to re-contextualize and re-tool research. Also active methods of engagement and intervention such as actionresearch and design ethnographies [49] seem to be a viable alternative to re-tool theories in equilibrium periods. New alternative methods of inquiry can be used as well; "we can leverage our analytics on big data (both qualitative and quantitative) to discover patterns that are then abstracted into broader theories." [61, p. 32]. We speculate big data analytics can be an important tool to assess punctuations and re-tool theories used to explain constant changing phenomenon.

\section{Concluding Comments}

Unstable phenomena have many characteristics that can be used to recognize them; they are dynamic, may seem to have unpredictable variables and relationships, and include events that challenge researchers' ability to provide consistent findings and accumulation of knowledge over time. In IS, many phenomena can be considered unstable. Such phenomena often show substantial instability as they first emerge, and then go through various punctuations over time, alternating between instability and stability. As described earlier in the paper, instability might explain the changes in a given IS phenomenon at different points in time when different punctuations might have affected the given phenomenon. We discussed two subfields within IS that demonstrate instability: word processing, and information security. The word processing example allowed us to show that instability can be found in most if not all IS domains, 
whereas the InfoSec example allowed us to provide a deeper discussion of unstable phenomena.

It is important for researchers to recognize the inherent dynamic nature of many of the environmental factors they investigate in the IS discipline. We therefore proposed a simple framework to re-think how we deal with unstable phenomena, the R3 framework. We argue that the most crucial " $R$ " is to recognize the phase of stability or instability of a focal phenomenon. Without such recognition, researchers are unlikely to consider re-contextualizing and retooling. As researchers recognize instability, they may reconsider prior findings while they conduct their literature reviews or meta-analyses with the lens of instability, which could explain how the object of study has changed.

The latter two elements of the framework, while presented separately in the previous section, affect each other. The need to re-contextualize often goes hand in hand with the need to retool or re-consider the methodological approaches that can be used. For example, we discussed phenomenon immersion (indepth involvement in the phenomenon of interest) as one approach to help properly re-contextualize and retheorize for various periods of instability of a target phenomenon. We add our voice to the calls for more longitudinal studies in IS phenomena, focusing on the need to understand the evolution of a phenomenon through its various phases of stability and instability.

It is also important to note that events like those related to the COVID-19 pandemic may create a period of temporary instability. Data currently being collected by researchers may have a bias due to this contextual instability. Research that utilizes secondary data may have to handle data during this pandemic differently (i.e., mark as an anomaly) due to the pandemic situation. In other situations, researchers may be able to use this pandemic as a point in time that triggered a change in punctuation and necessitates a different theoretical investigation of a phenomenon.

\section{References}

[1] Lyytinen, K., and Newman, M., "Explaining Information Systems Change: A Punctuated Socio-Technical Change Model", European Journal of Information Systems, 17(6), 2008, pp. 589613.

[2] Sidorova, A., Evangelopoulos, N., Valacich, J.S., and Ramakrishnan, T., "Uncovering the Intellectual Core of the Information Systems Discipline", MIS Quarterly, 32(3), 2008, pp. 467-A420.

[3] Grover, V., Carter, M., and Jiang, D., "Trends in the Conduct of Information Systems Research", Journal of Information Technology, 34(2), 2019, pp. 160-177.

[4] Baskerville, R., Myers, M., and Yoo, Y., "Digital First: The Ontological Reversal and New Challenges for Is Research", 2019, [5] Menard, P., Bott, G.J., and Crossler, R.E., "User Motivations in Protecting Information Security: Protection Motivation Theory Versus Self-Determination Theory", Journal of Management Information Systems, 34(4), 2017, pp. 1203-1230.
[6] D'Arcy, J., and Herath, T., "A Review and Analysis of Deterrence Theory in the Is Security Literature: Making Sense of the Disparate Findings", European Journal of Information Systems, 20(6), 2011, pp. 643-658.

[7] Crossler, R.E., and Bélanger, F., "The Quest for Complete Security Protection: An Empirical Analysis of an Individual's 360 Degree Protection from File and Data Loss", 18th Americas Conference on Information Systems, 2012

[8] Gupta, S., and Bostrom, R.P., "A Revision of Computer SelfEfficacy Conceptualizations in Information Systems", SIGMIS Database, 50(2), 2019, pp. 71-93.

[9] Marakas, G., Johnson, R., and Clay, P.F., "The Evolving Nature of the Computer Self-Efficacy Construct: An Empirical Investigation of Measurement Construction, Validity, Reliability and Stability over Time", Journal of the Association for Information Systems, 8(1), 2007, pp. 2.

[10] Faulkner, P., and Runde, J., "Theorizing the Digital Object", MIS Quarterly, 43(4), 2019,

[11] Boss, S.R., Galletta, D.F., Lowry, P.B., Moody, G.D., and Polak, P., "What Do Systems Users Have to Fear? Using Fear Appeals to Engender Threats and Fear That Motivate Protective Security Behaviors", MIS Quarterly, 39(4), 2015, pp. 837-864.

[12] Truex, D., Holmström, J., and Keil, M., "Theorizing in Information Systems Research: A Reflexive Analysis of the Adaptation of Theory in Information Systems Research", Journal of the Association for Information Systems, 7(12), 2006, pp. 797821.

[13] Gould, N.E.-S.J., and Eldredge, N., "Punctuated Equilibria: An Alternative to Phyletic Gradualism", Essential readings in evolutionary biology, 1972, pp. 82-115.

[14] Hassan, N.R., "Is Information Systems a Discipline? Foucauldian and Toulminian Insights", European Journal of Information Systems, 20(4), 2011, pp. 456-476.

[15] Westland, J.C., "The Is Core Xii: Authority, Dogma, and Positive Science in Information Systems Research", Communications of the Association for Information Systems, 13(1), 2004, pp. 12.

[16] Applegate, L.M., "E-Business Models: Making Sense of the Internet Business Landscape", in (Dickson, G., Gary, W., and Desanctis, G., 'eds.'): Information Technology and the Future Enterprise: New Models for Managers, Prentice Hall, Upper Saddle River, NJ, 2001

[17] vom Brocke, J., Simons, A., Riemer, K., Niehaves, B., Plattfaut, R., and Cleven, A., "Standing on the Shoulders of Giants: Challenges and Recommendations of Literature Search in Information Systems Research", CAIS, 37(1), 2015, pp. 205-224. [18] Baskerville, R.L., and Myers, M.D., "Fashion Waves in Information Systems Research and Practice", MIS Quarterly, 33(4), 2009, pp. 647-662.

[19] Gill, G., and Bhattacherjee, A., "Fashion Waves Versus Informing: Response to Baskerville and Myers", MIS Quarterly, 33(4), 2009, pp. 667-671.

[20] Cram, W.A., Brohman, M.K., and Gallupe, R.B., "Hitting a Moving Target: A Process Model of Information Systems Control Change", Information Systems Journal, 26(3), 2016, pp. 195-226. [21] Preston, D., and Chen, D., "From Exploitation to Exploration: A Staged Model of Supplier Information Technology Use for Buyer Value Creation", Proceedings of the 52nd Hawaii International Conference on System Sciences 2019

[22] Sabherwal, R., Hirschheim, R., and Goles, T., "The Dynamics of Alignment: Insights from a Punctuated Equilibrium Model", Organization Science, 12(2), 2001, pp. 179-197.

[23] Ceruzzi, P.E., and Grad, B., "Guest Editors' Introduction: Pc Software--Word Processing for Everyone", Annals of the History of Computing, IEEE, 28(4), 2006, pp. 4-5. 
[24] Bergin, T.J., "The Origins of Word Processing Software for Personal Computers: 1976-1985", IEEE Annals of the History of Computing, 28(4), 2006, pp. 32-47.

[25] Bergin, T.J., "The Proliferation and Consolidation of Word Processing Software: 1985-1995", IEEE Annals of the History of Computing, 28(4), 2006, pp. 48-63.

[26] Merschbrock, C., and Munkvold, B.E., "Effective Digital Collaboration in the Construction Industry - a Case Study of Bim Deployment in a Hospital Construction Project", Computers in Industry, 73(2015, pp. 1-7.

[27] Kumaran, K., "Synchronous and Asynchronous Collabrative Writing Methods on the Cloud", in (Editor, 'ed.'^'eds.'): Book Synchronous and Asynchronous Collabrative Writing Methods on the Cloud, SRM University, Digital Library, 2012

[28] McLeod Jr., R., and Bender, D.H., "The Integration of Word Processing into a Management Information System", MIS Quarterly, 6(4), 1982, pp. 11-29.

[29] West, T.W., and Mullins, C.J., "Word Processing in a Major Corporation: Policies, Plans, and Management", MIS Quarterly, 5(4), 1981, pp. 1-18.

[30] Langone, J., and Willis, C., "Effects of Computer-Based Word Processing Versus Paper/Pencil Activities on the Paragraph", Journal of Research on Computing in Education, 27(2), 1994, pp. 171.

[31] Kukich, K., "Techniques for Automatically Correcting Words in Text", ACM Computing Surveys, 24(4), 1992, pp. 376439.

[32] Garfinkel, R., Fernandez, E., and Gopal, R., "Design of an Interactive Spell Checker: Optimizing the List of Offered Words", Decision Support Systems, 35(3), 2003, pp. 385-397.

[33] Dewan, R.M., and Freimer, M.L., "Consumers Prefer Bundled Add-Ins", Journal of Management Information Systems, 20(2), 2003, pp. 99-111.

[34] Ceaparu, I., Lazar, J., Bessiere, K., Robinson, J., and Shneiderman, B., "Determining Causes and Severity of End-User Frustration", International Journal Of Human-Computer Interaction, 17(3), 2004, pp. 333-356.

[35] Lazar, J., Jones, A., and Shneiderman, B., "Workplace User Frustration with Computers: An Exploratory Investigation of the Causes and Severity", Behaviour \& Information Technology, 25(3), 2006, pp. 239-251.

[36] Chakravarty, S., Dogan, K., and Tomlinson, N., "A Hedonic Study of Network Effects in the Market for Word Processing Software", Decision Support Systems, 41(4), 2006, pp. 747-763.

[37] Roberts, C., "Biometric Attack Vectors and Defences", Computers \& Security, 26(1), 2007, pp. 14-25.

[38] Crossler, R., Johnston, A.C., Lowry, P.B., Hu, Q., Warkentin, M., and Baskerville, R., "Future Directions for Behavioral Information Security Research", Computers \& Security, 32(February), 2013, pp. 90-101.

[39] Bakshi, A., Dixit, V., and Mehta, K., "Virus: A Menace for Information Security", Global Journal of Enterprise Information System, 2(1), 2010, pp. 58-70.

[40] Fosnock, C., "Computer Worms: Past, Present, and Future", East Carolina University, 8(2005, pp. 1-9.

[41] http://www.foxnews.com/tech/2012/01/26/dont-use-ourproduct-symantec-warns-customers.html, accessed February 14, 2018.

[42] Lévesque, F.L., Chiasson, S., Somayaji, A., and Fernandez, J.M., "Technological and Human Factors of Malware Attacks: A Computer Security Clinical Trial Approach", ACM Transactions on Privacy and Security, 21(4), 2018, pp. 1-30.

[43] Crossler, R.E., "Protection Motivation Theory: Understanding Determinants to Backing up Personal Data", 43rd Hawaii International Conference on System Sciences (HICSS), 2010, pp. 1-10.
[44] Herath, T., and Rao, H., "Protection Motivation and Deterrence: A Framework for Security Policy Compliance in Organisations", European Journal of Information Systems, 18(2), 2009, pp. 106-125.

[45] Johnston, A.C., and Warkentin, M., "Fear Appeals and Information Security Behaviors: An Empirical Study", MIS Quarterly, 34(3), 2010, pp. 548-566.

[46] Lee, Y., and Larsen, K.R., "Threat or Coping Appraisal: Determinants of Smb Executive's Decision to Adopt AntiMalware Software", European Journal of Information Systems, 18(2), 2009, pp. 177-187.

[47] Johns, G., "The Essential Impact of Context on Organizational Behavior", Academy of Management Review, 31(2), 2006, pp. 386-408.

[48] Whetten, D.A., "An Examination of the Interface between Context and Theory Applied to the Study of Chinese Organizations", Management and Organization Review, 5(1), 2009, pp. 29-55.

[49] Baskerville, R., and Myers, M., "Design Ethnography in Information Systems", Information Systems Journal, 25(1), 2015, pp. 23-46.

[50] Crossler, R.E., Di Gangi, P.M., Johnston, A.C., Bélanger, F., and Warkentin, M., "Providing Theoretical Foundations: Developing an Integrated Set of Guidelines for Theory Adaptation", Communications of the Association for Information Systems, 43(1), 2018, pp. 31.

[51] Uotila, J., "Punctuated Equilibrium or Ambidexterity: Dynamics of Incremental and Radical Organizational Change over Time", Industrial and Corporate Change, 27(1), 2017, pp. 131-148.

[52] Hong, W., Chan, F.K.Y., Thong, J.Y.L., Chasalow, L.C., and Dhillon, G., "A Framework and Guidelines for Context-Specific Theorizing in Information Systems Research", Information Systems Research, 25(1), 2014, pp. 111-136.

[53] Orlikowski, W.J., and Iacono, C.S., "Research Commentary: Desperately Seeking "It" in It Research - a Call to Theorizing the It Artifact", Information Systems Research, 12(2), 2001, pp. 121134.

[54] Anderson, C.L., and Agarwal, R., "Practicing Safe Computing: A Multimethod Empirical Examination of Home Computer User Security Behavioral Intentions", MIS Quarterly, 34(3), 2010, pp. 613-643.

[55] Jensen, M.L., Dinger, M., Wright, R.T., and Thatcher, J.B., "Training to Mitigate Phishing Attacks Using Mindfulness Techniques", Journal of Management Information Systems, 34(2), 2017, pp. 597-626.

[56] Gregor, S., "The Nature of Theory in Information Systems", MIS Quarterly, 30(3), 2006, pp. 611-642.

[57] MacKenzie, S.B., Podsakoff, P.M., and Podsakoff, N.P., "Construct Measurement and Validation Procedures in Mis and Behavioral Research: Integrating New and Existing Techniques", MIS Quarterly, 35(2), 2011, pp. 293-334.

[58] Catrambone, R., "Specific Versus General Procedures in Instructions", Human-Computer Interaction, 5(1), 1990, pp. 4993.

[59] Santhanam, R., and Wiedenbeck, S., "Modeling the Intermittent User of Word Processing Technology", Journal of the American Society for Information Science, 42(3), 1991, pp. 185196.

[60] Abetti, P.A., "The Birth and Growth of the Japanese Language Word Processor: Internal Venturing in Toshiba Corporation", International Journal of Technology Management, 18(1/2), 1999, pp. 114.

[61] Grover, V., "Surviving and Thriving in the Evolving Digital Age: A Peek into the Future of Is Research and Practice", ACM SIGMIS Database: the DATABASE for Advances in Information Systems, 50(1), 2019, pp. 25-34. 